\title{
Review \\ Pathological validation and significance of micrometastasis in sentinel nodes in primary breast cancer
}

\author{
Rajendra S Rampaul*, Ahmed Miremadi ${ }^{\dagger}$, Sarah E Pinder ${ }^{\dagger}$, Andrew $\mathrm{Lee}^{\dagger}$ and lan O Ellis ${ }^{\dagger}$
}

*Department of Surgery and ${ }^{*}$ Department of Pathology, Nottingham City Hospital, Nottingham, UK

Correspondence: Dr lan O Ellis, FRCPath, Reader in Pathology, City Hospital, Nottingham, Hucknall Road, Nottingham NG1 5PB, UK. Tel: +44 115969 1169; fax: +44 115962 7768; e-mail: ian.ellis@nottingham.ac.uk

Received: 12 December 2000

Accepted: 3 January 2001

Published: 23 January 2001
Breast Cancer Res 2001, 3:113-116

(C) 2001 BioMed Central Ltd

(Print ISSN 1465-5411; Online ISSN 1465-542X)

\begin{abstract}
In embracing a multidisciplinary approach to the management of patients with sentinel node biopsy in breast cancer, the pathologist task is to screen sentinel nodes for possible metastasis. The consequences of missing sentinel node micrometastasis can directly influence treatment strategies, and this screening therefore has to be performed with more attention than usual. There is presently great diversity in the histopathological work-up of sentinel nodes, with many centres employing additional techniques such as immunohistochemistry, reverse transcription polymerase chain reaction or flow cytometry in addition to routine haematoxylin and eosin staining. In this review, we address the pathological validation and significance of micrometastasis in sentinel node biopsy in primary breast cancer.
\end{abstract}

Keywords: micrometastasis, sentinel nodes

\section{Introduction}

The pathological methods used to assess axillary lymph node disease are of paramount importance because lymph node status is a powerful prognostic factor at the time of diagnosis. It has been shown through numerous studies that routine histological examination of dissected nodes may be inadequate depending on the thoroughness of examination [1,2]. Sapir and Amromin were, in 1948, the first to demonstrate this. They showed that, in 30 patients whose lymph nodes were reported as negative by standard histopathological methods using haematoxylin and eosin $(\mathrm{H} \& \mathrm{E})$, re-examination of multiple sections revealed 10 patients $(33 \%)$ had metastatic disease [1]. Pickren [2], working on lymph node specimens from 199 patients, similarly found that $22 \%$ of those free of disease on routine histopathological examination had occult metastasis using serial sectioning. The presence of such metastatic disease by serial sectioning has been shown to confer a significant adverse effect on recurrence and survival in some series. The major disadvantages, however, are the cost and labour implications in processing axillary lymph nodes through serial sectioning to search for occult disease.

Sentinel lymph node biopsy has rapidly emerged as a real surgical option in the management of primary breast cancer. The sensitivity of this method in axillary staging of breast cancer has been evaluated in numerous studies [3-10], and has been shown to depend on the pathological methods used to assess the sentinel lymph node. By reducing the number of lymph nodes to be studied, sentinel lymph node dissection makes more exhaustive histopathological examination possible. The optimal extent of the histopathological work-up is yet to be determined but, nonetheless, offers the possibility of examining lymph nodes in much greater detail without the drawbacks of cost and labour restrictions.

$\mathrm{H \& E}=$ haematoxylin and eosin; $\mathrm{IHC}=$ immunohistochemistry; $\mathrm{PCR}=$ polymerase chain reaction. 


\section{Pathological validation}

There is presently no consensus for the optimal handling of sentinel nodes in the laboratory. Most centres have developed their own 'in-house' protocols, which are invariably tied in with the institution's research ambitions. This ad hoc approach unfortunately can influence interpretation of results, as the amount of material being assessed between centres would be non-uniform even if we were to ignore methodological differences.

In a number of large studies, using different histopathologic techniques, the sentinel lymph node false negative rate (defined as how often the sentinel lymph node is negative for malignancy when cancer is present in the rest of the axilla) has varied between 0 and $11 \%$. For example, Giuliano et al [3] reported a false negative rate of $11 \%$ in a series of 174 patients. Their group obtained a frozen section intraoperatively and recommended taking permanent sections from at least two levels of the tissue block at $40 \mu \mathrm{m}$ intervals for H\&E staining and cytokeratin immunohistochemistry [4]. Krag et al [5] similarly reported a false negative rate of $11 \%$ in 405 patients. Lymph nodes were either bisected or sliced multiple times and one to three $H \& E$ sections were taken from the surface of each tissue block. Results of a more detailed examination of lymph nodes in 329 of these patients were reported by Weaver et al [6]. H\&E stained deeper sections at 100 and $200 \mu \mathrm{m}$ into the blocks, as well as cytokeratin immunohistochemical stains at $100 \mu \mathrm{m}$, were made and examined for all lymph nodes. The authors reported an overall decrease in residual axillary lymph node metastases from 13.3 to $11.1 \%$ after using deeper sections and immunohistochemical stains. None of the newly detected metastases were larger than $1 \mathrm{~mm}$, and more than $60 \%$ of them had a diameter of less than $0.1 \mathrm{~mm}$.

O'Hea et al [7], in a study of 55 patients at the Memorial Sloan-Kettering Cancer Centre, found a false negative rate of $13 \%$ using the routine pathological technique with a single section taken through each node. They reported using frozen section examination of the sentinel node for all patients with T1b or larger invasive cancers [8]. If the frozen section proved to be negative, the remainder of each sentinel lymph node is fixed and three additional sections are taken and stained by both H\&E and immunohistochemistry.

Veronesi et al [9] reported a false negative rate of $4.7 \%$ in a study of 163 patients and, in another study from the same centre, Viale et al [10] reported a false negative rate of $7 \%$ in 155 patients. All sentinel lymph nodes in the latter study were bisected and frozen. Fifteen pairs of adjacent frozen sections were cut at $50 \mu \mathrm{m}$ intervals (a total of 60 sections per lymph node). Additional pairs of sections were cut at $100 \mu \mathrm{m}$ intervals whenever residual tissue was left. One section of each pair was stained with H\&E and the other for cytokeratins. Non-sentinel lymph nodes were examined by standard histopathological techniques. Just $35.7 \%$ of positive nodes were detected on levels taken beyond the second pair of sections, and so would be missed if only one or two central sections were examined. All metastatic foci were identified on H\&E stained sections, although in three cases cytokeratin immunoreactivity was used to confirm the metastatic nature of cells. Viale et al [10] concluded that the intraoperative examination of axillary sentinel lymph nodes using serial sectioning is feasible and effective in predicting the axillary lymph node status.

A number of studies have tried, however, to address the question of optimal histopathologic examination of sentinel lymph nodes by comparison of different protocols performed on the same sample. Turner et al [4] examined 60 sentinel nodes, step sectioned at 10 levels separated by $40 \mu \mathrm{m}$, and stained by $\mathrm{H} \& \mathrm{E}$ and cytokeratin immunohistochemistry (IHC). Nine sentinel nodes (15\%) showed metastases in the first two levels, but study of levels 3-10 revealed only two additional metastases (3\%). In another study, Cserni [11] reported the results of serial sectioning and IHC staining of sentinel lymph nodes from 58 patients. Nodes were sectioned serially up to extinction of the blocks and every 10th to 20th level was examined. After every sixth section taken for H\&E, one was taken for IHC staining. The study showed that, in 8 out of 26 (31\%) positive nodes, the metastases could not be found if only one or two central sections were examined. IHC staining caused the detection of only two of these metastases. While Zhang et al [12] reported that taking three levels at approximately 25,50 and $75 \%$ of the tissue blocks discovered almost all metastases, the same strategy in the study of Cserni [11] would have missed $15 \%$ of the metastatic foci. van Diest [13] recommended, in an accompanying editorial, four additional levels at $250 \mu \mathrm{m}$ intervals with $\mathrm{H} \& \mathrm{E}$ and $\mathrm{IHC}$ staining when the first-level $\mathrm{H} \& \mathrm{E}$ section is negative.

A closer look at the literature interestingly reveals that the Italian group of Veronesi et al [9] do not 'lose' tissue to the molecular laboratory as do Guiliano et al [3]. The significance of interpreting results from such heterogeneous methodologies, where not all tissue is assessed histopathologically, is clearly highlighted in a recent paper by Dowlatshahi et al [14] who subjected the entire sentinel lymph node from 52 patients to analysis and found significant occult disease. Although the results of Dowlatshahi et al require prospective confirmation in larger studies, it nonetheless emphasises the need for uniformity in examining sentinel lymph nodes.

We at Nottingham City Hospital employ the MRC ALMANAC protocol to assess all SNB. These nodes are cut into slices about $3 \mathrm{~mm}$ thick, taken perpendicular to the long axis to maximise the assessment of the marginal sinus, with one node per cassette. We have found in our experi- 
ence that the majority of nodes can be completely embedded in one cassette. Larger nodes have alternate slices embedded and may require more than one cassette. Very large obviously involved nodes have one section taken. This approach is consistent with recommendations of the National Health Service Breast Screening Programme [15].

Some authors have advocated intraoperative frozen sections or imprint cytology of axillary lymph nodes. Conventional frozen sections, we believe, have an unacceptably high false negative rate of 10-30\% [16-19]. More intensive intraoperative assessment with serial sections and immunohistochemistry has been described, but this is time consuming and labour intensive. A frozen section might be appropriate in selected cases; for example, if the node is macroscopically abnormal and this is confirmed histologically to be metastatic carcinoma, further axillary surgery can be performed immediately. Some studies have found low false negative rates of $2-3 \%$ with intraoperative imprint cytology $[16,20]$, but not all have been able to achieve this level of accuracy [18]. Most breast carcinomas can be diagnosed preoperatively, and we extremely rarely perform frozen section diagnosis of the primary tumour. Our feeling is that frozen section and imprint cytology assessment of axillary nodes is also inappropriate.

Reverse transcription polymerase chain reaction (PCR) is even more sensitive than immunohistochemistry at detecting metastatic tumour $[21,22]$. Two types of method can be used to detect tumour cells with molecular techniques. Firstly, a genetic defect such as chromosomal rearrangement or mutation can be identified. The problem with this method is that no genetic defect is seen in all breast carcinomas. The second method is based on a molecular marker that is transcribed by tumour cells, but not by the adjacent tissue. To obtain a marker that has this specificity and is expressed in the majority of tumours is difficult. It may be necessary to use a panel of markers. A major problem with PCR is a high false positive rate due to the sensitivity of the method. It is also not possible with PCR to determine whether the DNA comes from a viable cell. An advantage of $\mathrm{H} \& \mathrm{E}$ sections and IHC is that the morphology of the cells can be examined and malignancy confirmed. A major unresolved question is whether carcinoma detected only by PCR is of prognostic significance.

\section{Clinical significance of micrometastasis}

There is no universally agreed definition of micrometastasis. Huvos et al defined micrometastasis as metastatic foci less than $2 \mathrm{~mm}$ in diameter [23] and this is still widely used. In fact, the fifth edition of the AJCC staging manual uses the same definition; however, the arbitrary cutoff point varies between 0.2 and $2 \mathrm{~mm}$ in various studies.

The detection rate of micrometastases in axillary lymph nodes has been reported to range from 9 to 46\% [14,24].
Studies have used serial sectioning with or without immunohistochemical stains for the detection of micrometastatic foci and, as described, these methods have been shown to have a definite impact on detection rates.

Despite all this, the clinical significance of micrometastasis is yet to be determined. Nodal micrometastases appear to have a small but significant adverse effect on distant recurrence and survival in some studies, but not in all [16]. It is imperative that the prognostic significance of nodal micrometastases be determined, as there are important implications for systemic adjuvant chemotherapy and hormonal therapy. The International (Ludwig) Breast Cancer Study [25], one of the largest micrometastases studies performed to date, showed that both disease free interval and overall survival were significantly affected by the presence of micrometastatic disease at 5 and 6 years of followup. At 6 years median follow-up, DFS was reduced from $71 \%$ in the node negative group to $53 \%$ in micrometastatic node positive patients $(P=0.0008)$. The overall survival was $80 \%$ in the node negative group and $70 \%$ in the node positive group $(P=0.0009)$ [26]. Rosen et al found that patients had poorer disease free interval and overall survival at 10 years with nodal metastasis smaller than $2 \mathrm{~mm}$. However, this described a group with T1 tumours, whereas no survival disadvantage as such was found in T2 cancer [27]. Present-day data of sentinel lymph node micrometastases do not possess sufficient follow-up to observe any survival effects as some retrospective studies have shown, and therefore the prognostic significance of such occult metastasis at this time is unknown.

\section{Conclusion}

The traditional 'Halsted' view of breast cancer was that there was a logical sequential pattern of spread of the carcinoma, and this was used to justify radical surgery. Fisher et al [28] and other workers have more recently proposed that carcinoma of the breast is often disseminated at the time of diagnosis, and that nodal metastases are merely a marker of systemic disease. There is a trend towards less radical surgery to both the breast and the axilla associated with this philosophical change. Axillary sentinel lymph node biopsy is thus a reversion to the 'Halsted' philosophy, while at the same time continuing the trend towards less radical surgery. In reality, the truth probably lies somewhere between the 'Halsted' and 'Fisher' models. Two central questions particularly require answers from clinical trials: whether patients with a negative sentinel axillary lymph node(s) can safely avoid further axillary surgery, and the clinical significance of micrometastases. Of critical importance to both these questions is the way in which the pathologist should assess the node biopsy, and thus how negative and positive nodes are defined. The cost in time, equipment and consumables needs to be compared for sentinel node biopsy and other forms of axillary surgery. Comparison of the effectiveness of sentinel node biopsy 
with axillary node sampling also needs to be addressed, and this forms part of the UK ALMANAC study.

\section{References}

1. Sapir O, Amromin GD: Obscure axillary lymph node metastasis in carcinoma of the breast. Cancer 1948; 1:238-241.

2. Pickren JW: Significance of occult metastasis: a study of breast cancer. Cancer 1961; 14:1266-1271.

3. Giuliano AE, Kirgan DM, Guenther JM, Morton DL: Lymphatic mapping and sentinel lymphadenectomy for breast cancer. Ann Surg 1994; 220:391-398.

4. Turner RR, Ollila DW, Stern S, Giuliano AE: Optimal histopathologic examination of the sentinel lymph node for breast carcinoma staging. Am J Surg Pathol 1999; 23:263-267.

5. Krag D, Weaver D, Ashikaga T, Moffat F, Klimberg VS, Shriver C, Feldman S, Kusminsky R, Gadd M, Kuhn J, Harlow S, Beitsch P: The sentinel node in breast cancer - a multicenter validation study. N Engl J Med 1998; 339:941-946.

6. Weaver DL, Krag DN, Ashikaga T, Harlow SP, O'Connell M: Pathologic analysis of sentinel and nonsentinel lymph nodes in breast carcinoma: a multicenter study. Cancer 2000; 88: 1099-1107.

7. O'Hea BJ, Hill AD, El-Shirbiny AM, Yeh SD, Rosen PP, Coit DG, Borgen PI, Cody HS III: Sentinel lymph node biopsy in breast cancer: initial experience at Memorial Sloan-Kettering Cancer Center. J Am Coll Surg 1998; 186:423-427.

8. Cody HS III, Borgen PI: State-of-the-art approaches to sentinel node biopsy for breast cancer: study design, patient selection, technique, and quality control at Memorial Sloan-Kettering Cancer Center. Surg Oncol 1999; 8:85-91.

9. Veronesi U, Paganelli G, Galimberti V, Viale G, Zurrida S, Bedoni M, Costa A, de Cicco C, Geraghty JG, Luini A, Sacchini V, Veronesi $P$ : Sentinel-node biopsy to avoid axillary dissection in breast cancer with clinically negative lymph nodes. Lancet 1997; 349:1864-1867.

10. Viale G, Bosari S, Mazzarol G, Galimberti V, Luini A, Veronesi P, Paganelli G, Bedoni M, Orvieto E: Intraoperative examination of axillary sentinel lymph nodes in breast carcinoma patients. Cancer 1999; 85:2433-2438.

11. Cserni G: Metastases in axillary sentinel lymph nodes in breast cancer as detected by intensive histopathological work up. J Clin Pathol 1999; 52:922-924.

12. Zhang PJ, Reisner RM, Nangia R, Edge SB, Brooks JJ: Effectiveness of multiple-level sectioning in detecting axillary nodalmicrometastasis in breast cancer: a retrospective study with immunohistochemical analysis. Arch Pathol Lab Med 1998; 122:687-690.

13. van Diest PJ: Histopathological workup of sentinel lymph nodes: how much is enough? J Clin Pathol 1999; 52:871-873.

14. Dowlatshahi K, Fan M, Bloom KJ, Spitz DJ, Patel S, Snider HC Jr: Occult metastases in the sentinel lymph nodes of patients with early stage breast carcinoma: a preliminary study. Cancer 1999; 86:990-996.

15. National Coordinating Group for Breast Screening Pathology: Pathology Reporting in Breast Cancer Screening. Sheffield: National Health Service Breast Screening Programme, 1995.

16. Fisher CJ, Boyle S, Burke M, Price AB: Intraoperative assessment of nodal status in the selection of patients with breast cancer for axillary clearance. Br J Surg 1993; 80:457-458.

17. Galimberti V, Zurrida S, Zucali $P$, Luini A: Can sentinel node biopsy avoid axillary dissection in clinically node-negative breast cancer patients? Breast 1998; 7:8-10.

18. van Diest PJ, Torrenga $H$, Borgstein PJ, Pijpers R, Bleichrodt RP Rahusen FD, Meijer S: Reliability of intraoperative frozen section and imprint cytological investigation of sentinel lymph nodes in breast cancer. Histopathology 1999; 35:14-18.

19. Veronesi U, Paganelli G, Viale G, Galimberti V, Luini A, Zurrida S, Robertson C, Sacchini V, Veronesi P, Orvieto E, De Cicco C, Intra M, Tosi G, Scarpa D: Sentinel lymph node biopsy and axillary dissection in breast cancer: results in a large series. J Natl Cancer Inst 1999; 91:368-373.

20. Rubio IT, Korourian S, Cowan C, Krag DN, Colvert M, Klimberg VS: Sentinel lymph node biopsy for staging breast cancer. Am J Surg 1998; 176:532-535.

21. Noguchi S, Aihara T, Nakamori S, Motomura K, Inaji H, Imaoka S, Koyama $\mathrm{H}$ : The detection of breast carcinoma micrometas- tases in axillary lymph nodes by means of reverse transcriptase-polymerase chain reaction. Cancer 1994; 74:1595-1160.

22. Schoenfeld A, Luqmani Y, Smith D, O'Reilly S, Shousha S, Sinnett HD, Coombes RC: Detection of breast cancer micrometastases in axillary lymph nodes by using polymerase chain reaction. Cancer Res 1994; 54:2986-2990.

23. Huvos AG, Hutter RV, Berg JW: Significance of axillary macrometastases and micrometastases in mammary cancer. Ann Surg 1971; 173:44-46.

24. Dowlatshahi K, Fan M, Snider HC, Habib FA: Lymph node micrometastases from breast carcinoma: reviewing the dilemma. Cancer 1997; 80:1188-1197.

25. International (Ludwig) Breast Cancer Study Group: Prognostic importance of occult axillary lymph node micrometastases from breast cancers. Lancet 1990; 335:1565-1568.

26. Neville AM, Price KN, Gelber RD, Goldhirsch A: Axillary node micrometastases and breast cancer [letter]. Lancet 1991; 337: 1110.

27. Rosen PP, Groshen S, Saigo PE, Kinne DW, Hellman S: A long term follow up study of survival in stage I (T1NOMO) and stage II (T1NOMO) breast carcinoma. J Clin Oncol 1989; 7:355-366.

28. Fisher B, Wolmark N, Bauer M, Redmond C, Gebhardt M: The accuracy of clinical nodal staging and of limited axillary dissection as a determinant of histologic nodal status in carcinoma of the breast. Surg Gynecol Obstet 1981; 152:765-772. 